\title{
Visualization of High Throughput Genomic Data Using R and Bioconductor
}

Ruchi Yadav and Prachi Srivastava*

Amity Institute of Biotechnology, Amity University, Uttar Pradesh, Lucknow, India

\begin{abstract}
DNA microarrays, technology aims at the measurement of mRNA levels in particular cells or tissues for many genes simultaneously. Microarray in molecular biology results in huge datasets that need rigorous computational analysis to extract biological information that lead to some conclusion. From printing of microarray chip to hybridization and scanning process it results in variability in quality of data due to which actual information is either lost or it is over represented. Computational analysis plays an important part related to the processing of the biological information embedded in microarray results and for comparing gene expression result obtained from different samples in different condition for biological interpretation. A basic, yet challenging task is quality control and visualization of microarray gene expression data. One of the most popular platforms for microarray analysis is Bioconductor, an open source and open development software project for the analysis and comprehension of genomic data, based on the $\mathrm{R}$ programming language. This paper describes specific procedures for conducting quality assessment of Affymetrix Gene chip using data from GEO database GSE53890 and describes quality control packages of bioconductor with reference to visualization plots for detailed analysis. This paper can be helpful for any researcher working on microarray analysis for quality control analysis of affymetrix chip along with scientific interpretations.
\end{abstract}

Keywords: Microarray; R; Bioconductor; Transcriptomics; Quality control; Genome visualization

\section{Introduction}

In the context of the human genome project, new technologies emerged that facilitate the parallel execution of experiments on a large number of genes simultaneously. The measurement of transcriptional activity in living cells is of fundamental importance in many fields of research from basic biology to the study of complex diseases such as cancer [1]. The so-called DNA microarrays, or DNA chips, constitute a prominent example. This technology aims at the measurement of mRNA levels in particular cells or tissues for many genes at once [2]. DNA microarrays provide an instrument for measuring the mRNA abundance of tens of thousands of genes. Currently, the measurements are based on mRNA from samples of hundreds to millions of cells, thus expression estimates provide an ensemble average of a possibly heterogeneous population [3].

Gene expression profiling provides unprecedented opportunities to study patterns of gene expression regulation, for example, in diseases or developmental processes. DNA microarray technology takes advantage of hybridization properties of nucleic acid and uses complementary molecules attached to a solid surface, referred to as probes single strands of complementary DNA for the genes of interestwhich can be many thousands are immobilized on spots arranged in a grid (array) on a support which will typically be a glass slide, a quartz wafer, or a nylon membrane. From a sample of interest, e.g. a tumor biopsy, the mRNA is extracted, labeled and hybridized to the array [4]. Measuring the quantity of label on each spot then yields an intensity value that should be correlated to the abundance of the corresponding RNA transcript in the sample [5]. Microarrays provide a rich source of data on the molecular working of cells. Each microarray reports on the abundance of tens of thousands of mRNAs. Virtually every human disease is being studied using microarrays with the hope of finding the molecular mechanisms of disease [6-8].

Bioinformatics analysis plays an important part of processing the information embedded in large-scale expression profiling studies and for laying the foundation for biological interpretation [8-10].
A basic, yet challenging task in the analysis of microarray gene expression data is the identification of changes in gene expression that are associated with particular biological conditions. Careful statistical design and analysis are essential to improve the efficiency and reliability of microarray experiments throughout the data acquisition and analysis process [11-12].

\section{Microarray Studies}

Microarrays are useful in a wide variety of studies with a wide variety of objectives. Many of these objectives fall into the following categories $[13,14]$.

a) A typical microarray experiment is one who looks for genes deferentially expressed between two or more conditions. That is, genes which behave differently in one condition (for instance healthy [or untreated or wild type] cells) than in another (for instance tumor [or treated or mutant] cells). These are known as class comparison experiments.

b) When the emphasis is on developing a statistical model that can predict to which class a new individual belongs we have a class prediction problem. Examples of this are predicting the response to a treatment (e.g. classes are_responder_and_non-responder_) or the evolution of a disease (e.g. recidivated or cured).

c) Sometimes the objective is the identification of novel sub-types of individuals within a population. For example it has been shown

*Corresponding author: Prachi Srivastava, Assistant Professor, Departmen of Biotechnology, Amity University, Uttar Pradesh, Lucknow, India, Tel: +919453141916; E-mail: psrivastava@amity.edu; p.srivastava2@gmail.com

Received May 02, 2016; Accepted May 17, 2016; Published May 26, 2016

Citation: Yadav R, Srivastava P (2016) Visualization of High Throughput Genomic Data Using R and Bioconductor. J Data Mining Genomics Proteomics 7: 197. doi:10.4172/2153-0602.1000197

Copyright: ( 2016 Yadav R, et al. This is an open-access article distributed unde the terms of the Creative Commons Attribution License, which permits unrestricted use, distribution, and reproduction in any medium, provided the original author and source are credited. 
that certain types of leukemia present some subclasses that are very hard to distinguish morphologically but which can be classified using gene expression. This is an example of class discovery.

d) Pathway Analysis studies are those that try to find genes whose co regulation reflects their participation in common or related biochemical processes.

One of the most popular platforms for microarray analysis is Bioconductor, an open source and open development software project for the analysis and comprehension of genomic data, based on the $\mathrm{R}$ programming language. This paper describes specific procedures for conducting quality assessment of Affymetrix Gene Chip using data from GEO database using the open-source $\mathrm{R}$ programming environment in conjunction with the open-source Bioconductor software [15].

\section{Bioconductor and $\mathrm{R}$}

$R$ is a programming language. The name " $R$ " is initials of names of the two $\mathrm{R}$ authors (Robert Gentleman and Ross Ihaka). $\mathrm{R}$ is introduced in 1991 and R 1.0.0 is released in year 2000. Bioconductor emerges as a boon in life sciences and in high throughput experiments where analysis tools are available free of cost to analyze experimental data. In year 2008 Bioconductor version 2.4 is released and further follows $\mathrm{R}$ release. Current version of the Bioconductor is 3.2 and $\mathrm{R}$ version is 3.2.2 [16].

$\mathrm{R}$ environment is easy to use, coherent and have tools for data analysis. What make $\mathrm{R}$ different from other programming languages is its GUI for quick and easy upload of data along with tools for data manipulation, calculation and analysis along with it statistical tools facilitates calculation of standard deviation, variance, $\mathrm{t}$-test, $\mathrm{f}$-test and other statistical tools. R can be accessed from http://cran.r-project.org/ [17].

Bioconductor (www.bioconductor.org) provides bioinformatics tool for analyzing high throughput data that comes from experiment like microarray, SAGE, MS, MS-MS. Bioconductor data packages are divided into three categories Annotation Data, Experiment Data and Software. Currently there are 1104 software packages, 898 Annotation Data and 257 Experiment Data. It is very hard to identify particular package for set of experiments. This paper reviews the methods for visualization of affymetrix gene expression data. These steps are essential part of microarray data analysis that should be taken before utilized in processing and analysis of gene expression differential expression analysis [18].

Microarray data produces lots of experimental errors that emerged because of biasness in dye intensities, laser scanner, spotting errors, hybridization biasness. Before microarray analysis data must be cleaned and processed to extract biological information. Visualization and graphs representation are best suited to study microarray intensity files and comparing probe hybridization signals [19].

Evaluation of data quality is a major issue in microarray analysis. There are many packages that can be used for quality control analysis Table 1 lists the bioconductor packages that are used to study quality of chips and visualizing high throughput microarray data [20].

After quality control analysis and normalization differentially expressed genes calculation can be done for biological interpretation. Table 2 list the differentially expressed gene packages available at bioconductor [21].

\section{Materials and Methods}

The raw data for this study is retrieved from Gene Expression Omnibus database: http://www.ncbi.nlm.nih.gov/geo/; GEOID: GSE53890; http://

\begin{tabular}{|c|c|c|}
\hline S. No & Package & Description \\
\hline 1 & a4 & Automated Affymetrix Array Analysis \\
\hline 2 & a4Base & analysis of Affymetrix microarray experiments \\
\hline 3 & a4Core & Automated Affymetrix Array Analysis \\
\hline 4 & a4Preproc & package for preprocessing of microarray data \\
\hline 5 & a4Reporting & $\begin{array}{l}\text { Automated Affymetrix Array Analysis Reporting } \\
\text { Package }\end{array}$ \\
\hline 6 & affxparser & $\begin{array}{l}\text { Ackage for parsing Affymetrix files (CDF, CEL, CHP, } \\
\text { BPMAP, BAR) }\end{array}$ \\
\hline 7 & Affy & Exploratory oligonucleotide array analysis. \\
\hline 8 & affycomp & $\begin{array}{l}\text { Compare expression measures for Affymetrix } \\
\text { Oligonucleotide Arrays. }\end{array}$ \\
\hline 9 & affyContam & Affymetrix cel file data \\
\hline 10 & Affycoretools & Analyses with Affymetrix GeneChips \\
\hline 11 & AffyExpress & $\begin{array}{l}\text { Quality assessment and to identify differentially } \\
\text { expressed genes in the Affymetrix affymetrix chip }\end{array}$ \\
\hline 12 & Affyio & Parsing Affymetrix data files \\
\hline 13 & affylmGUI & $\begin{array}{l}\text { A Graphical User Interface for analysis of Affymetrix } \\
\text { microarray gene expression data using the affy and } \\
\text { limma Microarray packages }\end{array}$ \\
\hline 14 & affyPara & Oligonucleotide array analysis \\
\hline 15 & affyPLM & Quality assessment tools for affymetrix chip \\
\hline 16 & affyQCReport & Quality of a set of affymetrix arrays \\
\hline 17 & AffyRNADegradation & $\begin{array}{l}\text { Assessment and correction of RNA degradation } \\
\text { effects in Affymetrix } 3 \text { ' expression arrays }\end{array}$ \\
\hline 18 & AffyTiling & $\begin{array}{l}\text { Extraction and annotation of individual probes from } \\
\text { Affymetrix tiling arrays. }\end{array}$ \\
\hline 19 & annmap & Deep sequencing data analysis \\
\hline 20 & arrayQualityMetrics & Microarray quality metrics reports \\
\hline 21 & ArrayTools & $\begin{array}{l}\text { Quality assessment and to detect differentially } \\
\text { expressed genes for the Affymetrix GeneChips }\end{array}$ \\
\hline 22 & ExpressionView & $\begin{array}{l}\text { Isualizes possibly overlapping biclusters in a gene } \\
\text { expression matrix. }\end{array}$ \\
\hline 23 & limma & Linear Models for Microarray Data \\
\hline 24 & MiChip & $\begin{array}{l}\text { Microarray platform using locked oligonucleotides } \\
\text { for the analysis of the expression of microRNAs in } \\
\text { a variety of species }\end{array}$ \\
\hline 25 & Simpleaffy & High level analysis of Affymetrix data \\
\hline 26 & Starr & Affymetrix tiling arrays (ChIP-chip data) \\
\hline 27 & yaqcaffy & $\begin{array}{l}\text { Quality control of Affymetrix GeneChip expression } \\
\text { data }\end{array}$ \\
\hline 28 & annmap & $\begin{array}{l}\text { Genome annotation and visualisation package } \\
\text { pertaining to Affymetrix arrays }\end{array}$ \\
\hline
\end{tabular}

Table 1: Quality control packages of bio-conductor.

\begin{tabular}{|c|c|c|}
\hline $\begin{array}{l}\text { S. } \\
\text { No }\end{array}$ & $\begin{array}{l}\text { Name of } \\
\text { Package }\end{array}$ & Description \\
\hline 1 & DEGseq & $\begin{array}{l}\text { To identify differential expressed genes from rna-seq } \\
\text { data. }\end{array}$ \\
\hline 2 & DEGreport & Report of deg analysis. \\
\hline 3 & DEGraph & For two-sample test on graph. \\
\hline 4 & DEDS & $\begin{array}{l}\text { Differential expression via distance summary for } \\
\text { microarray data. }\end{array}$ \\
\hline 5 & DEseq2 & $\begin{array}{l}\text { Differential expression analysis based on negative } \\
\text { binomial distribution. }\end{array}$ \\
\hline 6 & DEXseq & For the inference of differential exon usage in rna-seq. \\
\hline 7 & Dexus & $\begin{array}{l}\text { For identifying the differential expression in rna-seq } \\
\text { studies with unknown conditions or without replicates. }\end{array}$ \\
\hline 8 & Derfinder & $\begin{array}{l}\text { Used for annotation-agnostic differential expression } \\
\text { analysis of rna-seq data at base-pair resolution. }\end{array}$ \\
\hline 9 & Derfinder plot & To find the plot for derfind. \\
\hline 10 & Diffbind & $\begin{array}{l}\text { Used for differential binding analysis of chip-seq peak } \\
\text { data. }\end{array}$ \\
\hline 11 & Diffgeneanalysis & Perform differential gene expression analysis. \\
\hline
\end{tabular}


Citation: Yadav R, Srivastava P (2016) Visualization of High Throughput Genomic Data Using R and Bioconductor. J Data Mining Genomics Proteomics 7: 197. doi:10.4172/2153-0602.1000197

www.ncbi.nlm.nih.gov/geo/query/acc.cgi?acc=GSE53890. GSE53890 is microarray experiment on REST and Stress Resistance in Aging and Alzheimer's disease (Figure 1) [22].

\section{Result and Conclusion}

From GSE53890 data out of 6 cel files are selected 3 females data and 3 males data chip used in this experiment is HG-U133_Plus_2. Gene expression file is created using affy package of bioconductor and visualization packages are used for quality control analysis and summarizing the output generated by these packages [23].

\section{Gene expression file}

Gene expression file created using affy package.

$$
\begin{aligned}
& >\text { library (affy) } \\
& >\text { array }=\text { ReadAffy (widget=TRUE) } \\
& >\text { eset }=\text { rma (array) } \\
& >\text { write.exprs (eset, file = "array.txt") }
\end{aligned}
$$

Figure 2 shows the gene expression file and expression values. Using above commands in $\mathrm{r}$ this file can be created and used for analysis either in $\mathrm{R}$ or in any other softwares like $\mathrm{MeV}$ that not accept .cel and .cdf file as input. Either object can be created for expression file using $>$ exprs=write.exprs (eset, file="array.txt")

Now this object exprs can be used directly in $r$ to visualize expression file and analyze the intensity.

\section{Visualization plots}

There are number of plots built in quality control packages for visualization of chips and analysis these plots are also used for comparing chips before and after normalization. Here we describe some plots and their analysis.

\section{Boxplot}

Boxplot also called as box-and-whisker plot is statistical plot that graphically plots numerical values for comparison of chips. Five values are plotted in box plot that are lower min value, lower quartile, mean, upper quartile, max value of chip files in parallel for comparison of chip intensities. Box plot display variation in sample without assuming any statistical distribution. Spacing between box lines indicates the spread or degree of dispersion in intensities values of one sample. Boxplot can also be created for individual variables in $\mathrm{R}$ using Boxplot function and using fivenum command we can access the five values of expression (Figure 3)

$$
\begin{aligned}
& \text { > library (affyQCReport) } \\
& \text { > affyQAReport (array) }
\end{aligned}
$$

Folder affyQA is created in same directory where $r$ working directory is set. This folder contains individual graph files.

\section{Intensity plot}

Intensity plot is similar to Boxplot but it gives more detailed view. Intensity plot $\mathrm{x}$-axis represents probe density and $\mathrm{y}$-axis probe intensity. Figure 4 represents the intensity plot between 6 cel files any array whose intensity graph is very different from other array is considered as problematic.

\section{RNA degradation plot}

RNA degradation plot is used to assess the quality of RNA molecule used as probe in chips. Since RNA probes are designed from 3' end of mRNA molecule because RNA degradation starts from 5' end of mRNA molecule so intensity should be less in 5 ' end as compared to 3'end of probe. This plot is used for quality analysis of probes spotted on affymetrix chip (Figure 5).

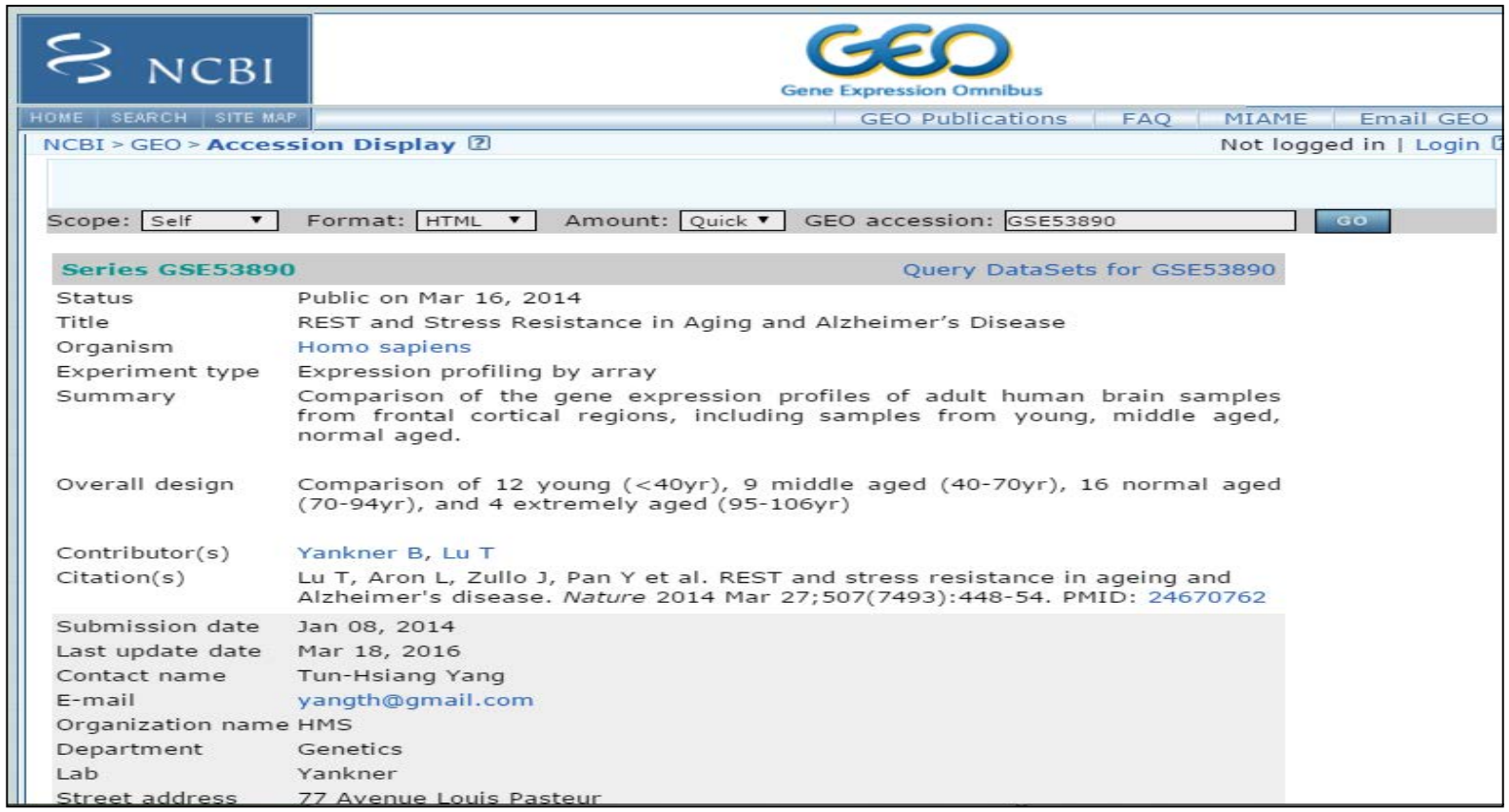

Figure 1: Gene expression Omnibus database. 
Citation: Yadav R, Srivastava P (2016) Visualization of High Throughput Genomic Data Using R and Bioconductor. J Data Mining Genomics Proteomics 7: 197. doi:10.4172/2153-0602.1000197

\begin{tabular}{|c|c|c|c|c|c|c|c|c|}
\hline 1 & A & B & c & D & E & F & G & H \\
\hline 1 & & 24 F.CEL & $26 \mathrm{M} . \mathrm{CEL}$ & 29 F.CEL & 34 F.CEL & $37 \mathrm{M} . \mathrm{CEL}$ & $40 \mathrm{M} . \mathrm{CEL}$ & \\
\hline 2 & 1007_s_at & 8.03429 & 8.772677 & 8.506786 & 8.806013 & 8.874959 & 8.471199 & \\
\hline 3 & 1053_at & 5.129433 & 5.26885 & 5.034688 & 5.031765 & 4.861491 & 4.917265 & \\
\hline 4 & 117_at & 4.944486 & 4.889163 & 4.710165 & 5.611009 & 4.827517 & 4.699408 & \\
\hline 5 & 121_at & 7.501474 & 7.355494 & 7.565741 & 7.450452 & 7.462104 & 7.602513 & \\
\hline 6 & 1255_g_at & 3.511665 & 3.580815 & 4.260613 & 3.928873 & 3.495896 & 3.578732 & \\
\hline 7 & 1294_at & 5.113248 & 5.258803 & 5.12377 & 4.937654 & 5.132642 & 5.137079 & \\
\hline 8 & 1316_at & 5.327564 & 5.341795 & 5.421229 & 5.412445 & 5.368434 & 5.43424 & \\
\hline 9 & 1320_at & 4.525333 & 4.445202 & 4.409657 & 4.461411 & 4.381585 & 4.396613 & \\
\hline 10 & 1405_i_at & 3.092261 & 2.880746 & 3.010714 & 3.030582 & 3.055263 & 3.075304 & \\
\hline 11 & 1431_at & 3.835525 & 3.752205 & 3.704723 & 4.03727 & 3.953105 & 3.936302 & \\
\hline 12 & 1438_at & 6.171631 & 5.769026 & 6.003033 & 5.839133 & 6.066168 & 5.910563 & \\
\hline 13 & 1487_at & 5.685933 & 5.872879 & 6.08433 & 6.100783 & 5.953952 & 6.146575 & \\
\hline 14 & 1494_ffat & 5.442937 & 5.490435 & 5.759878 & 5.539503 & 5.571146 & 5.545931 & \\
\hline 15 & 1552256_a & 6.993329 & 6.9624 & 6.836159 & 6.976911 & 7.118099 & 6.982972 & \\
\hline 16 & 1552257_a & 7.257667 & 7.068899 & 7.831783 & 7.312058 & 7.42301 & 7.59721 & \\
\hline 17 & 1552258_a & 3.781122 & 3.811404 & 3.816726 & 3.890403 & 3.699379 & 3.845689 & \\
\hline 18 & 1552261_a & 5.407248 & 4.970404 & 5.100554 & 5.080625 & 5.09663 & 5.054312 & \\
\hline 19 & 1552263_a & 2.968945 & 3.110073 & 3.036589 & 2.794043 & 3.069281 & 2.994463 & \\
\hline 20 & 1552264_e & 7.935421 & 7.499184 & 7.937578 & 7.256843 & 7.348546 & 6.982388 & \\
\hline 21 & 1552266_e & 3.375726 & 3.304053 & 3.527372 & 3.343631 & 3.184546 & 3.288351 & \\
\hline 22 & 1552269_a & 3.346049 & 3.267432 & 3.61881 & 3.190716 & 3.254016 & 3.181616 & \\
\hline 23 & 1552271_a & 4.683213 & 4.573254 & 4.539561 & 4.744894 & 4.597907 & 4.380566 & \\
\hline 24 & 1552272 a & 5.15859 & 4.690044 & 5.2258 & 4.978506 & 4.851615 & 5.034684 & \\
\hline 25 & 1552274_e & 5.627429 & 5.356057 & 6.119516 & 6.259067 & 6.417436 & 5.746686 & \\
\hline
\end{tabular}

Figure 2: The gene expression file and expression values.

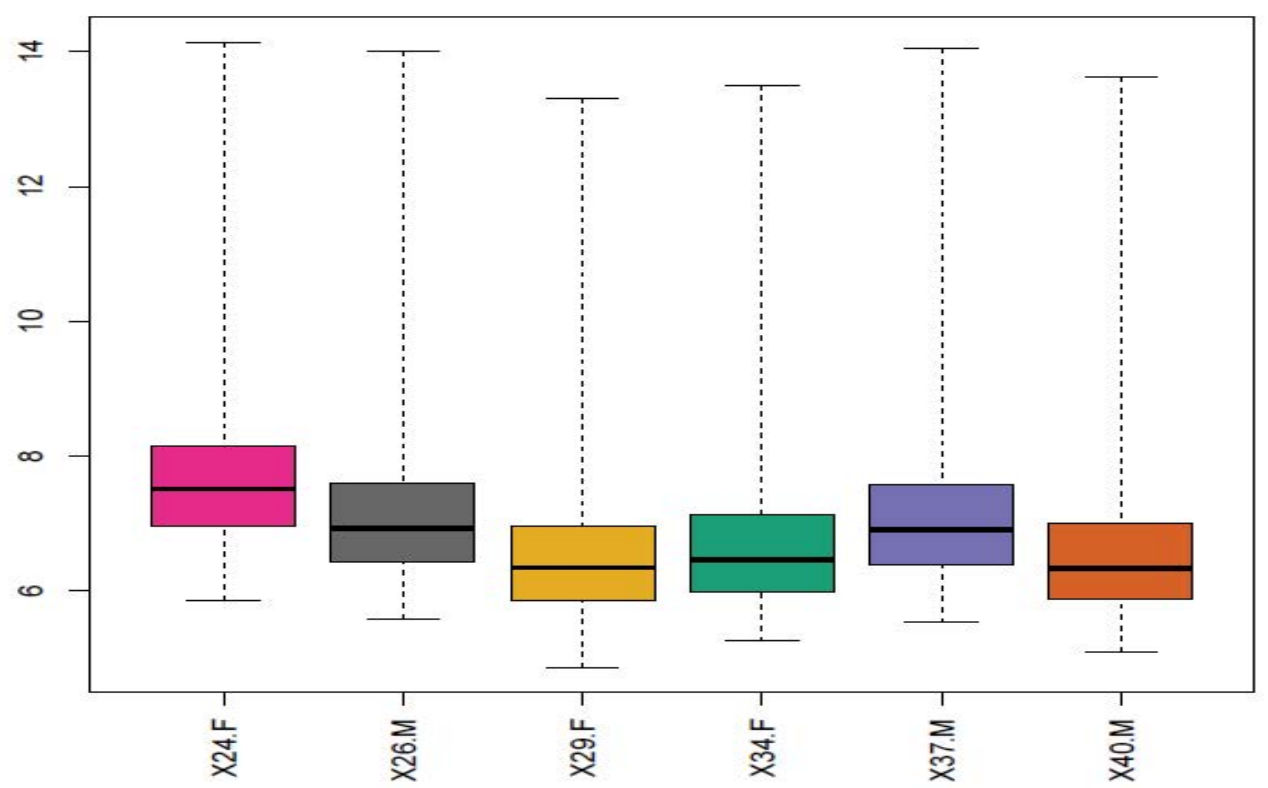

Figure 3: The Boxplot of gene expression file.

\section{MA plot}

MA plot where $\mathrm{M}$ represents minus sign and A represents mean addition sign for two channel microarray experiment.

Log Ratio: M ("Minus") = $\log _{2}(\mathrm{R} / \mathrm{G})=\log _{2} \mathrm{R}-\log _{2} \mathrm{G}$

Average Log Intensity: A (“Add”) $=\log _{2}(R G)^{1 / 2}$ or $(1 / 2)\left(\log _{2} R+\log _{2} G\right)$

MA plot is used to determine is there any biasness in intensities of red and green dye and other systemic errors or instrumental errors in experiment. To visualize the need of normalization before any analysis.
MA plot is plot of red and green intensities biasness and determine the rate of error in microarray experiment. Figure 6 represents the ma plot between. cel files and variances in intensities of red and green dyes.

\section{NUSE plot}

Normalized Unscaled Standard Errors (NUSE) is used for standard error for each probe on all chips. All probes are normalized to scale one across all arrays. This plot shows the variations between genes and any array with higher standard error is of poor quality and hence can be rejected for further analysis for biological interpretations Figure 7 shows the NUSE plot. 

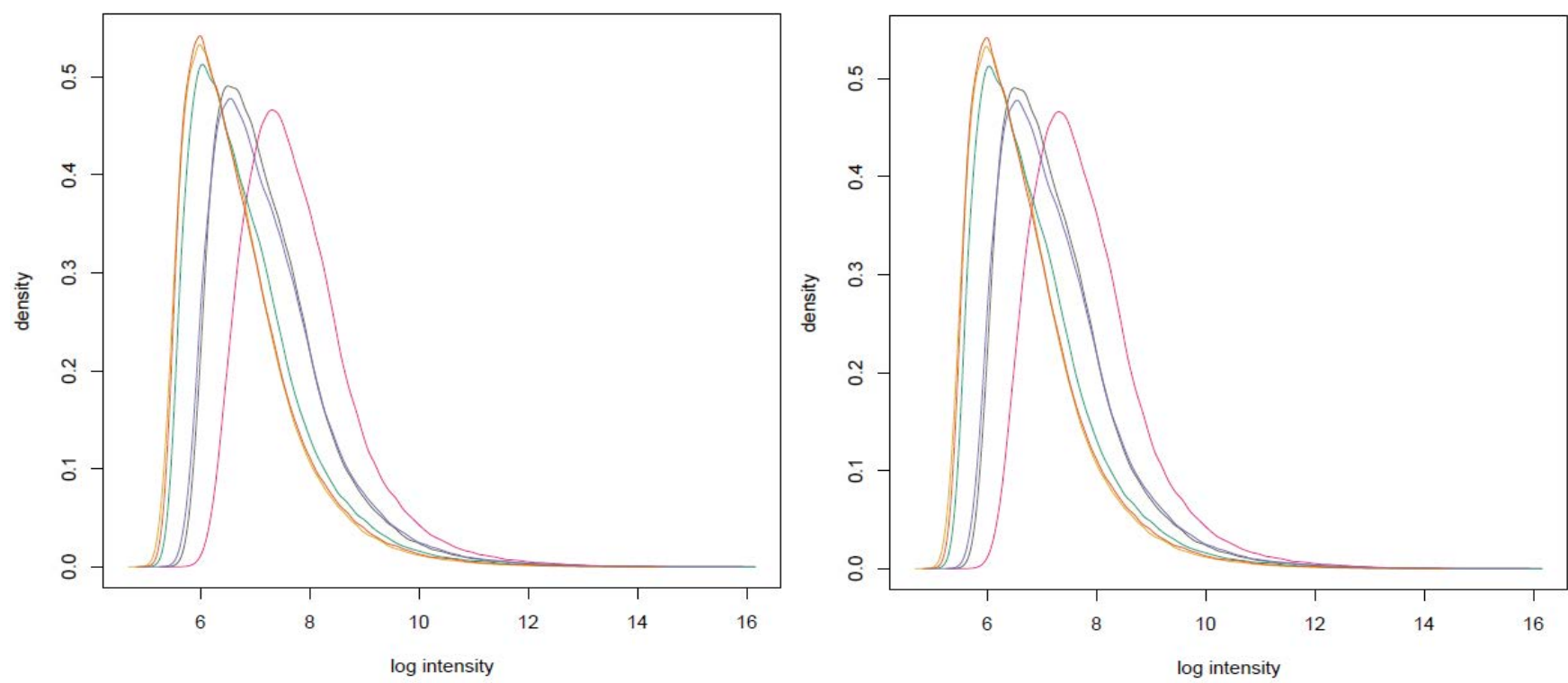

Figure 4: The intensity plot between 6 cel files any array.

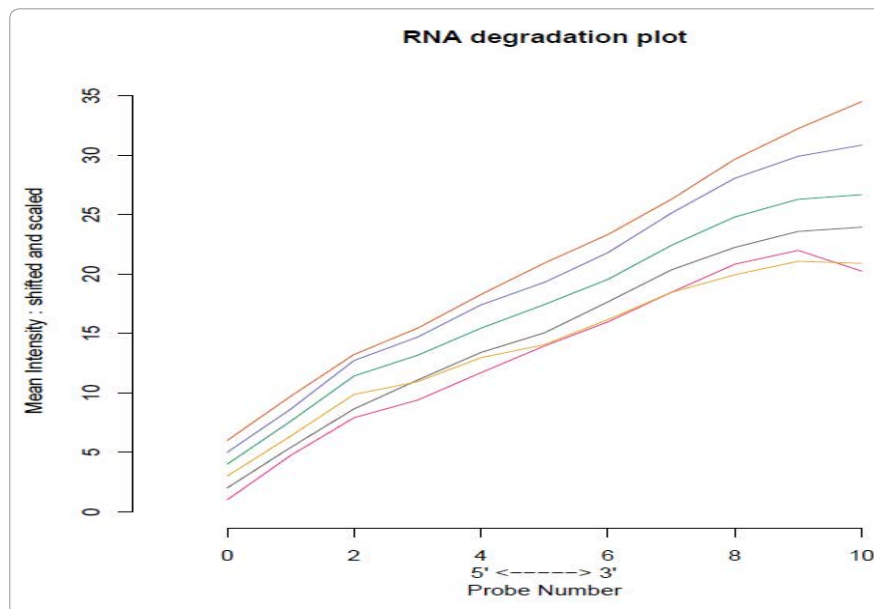

Figure 5: RNA degradation plot.

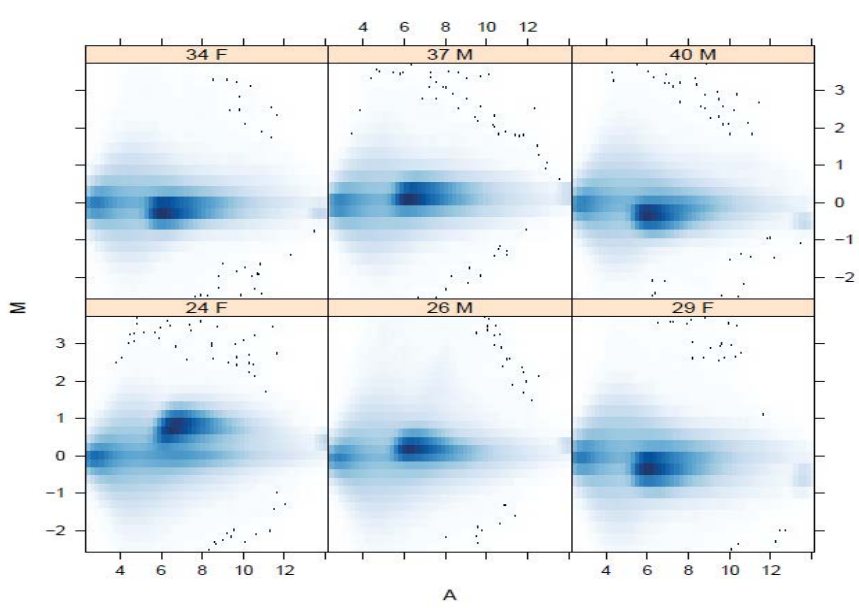

Figure 6: Ma plot between cel files and variances.

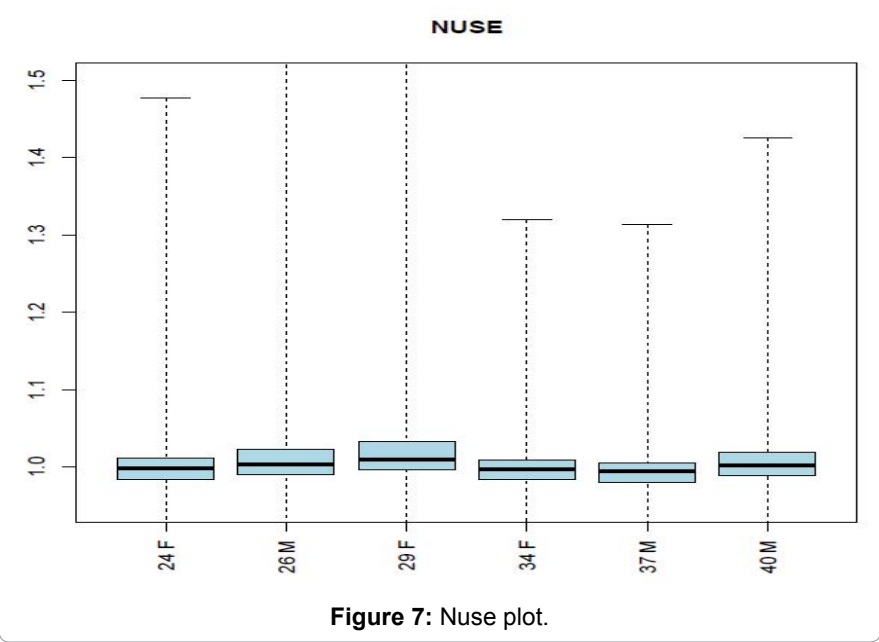

\section{RLE plot}

Relative Log Expression (RLE) plot is plot of RLE values that are calculated by assuming that all probeset across all array against median expression value for that probeset is constant and are not changing. Assuming that genes are constant across all arrays RLE values should be near 0.RLE plot is graphically plotted in form of Boxplot that provides quality of chips.

Figure 8 shows the RLE plot of chips in form of box plot and RLE values are set to 0 . This plot shows the variation in chips and used for quality assessment of probes.

\section{QC stats}

QC plot is recommended by affymetrix. Any array that is shown in red is indication of error and in blue indicates the array within limits of scale factors e within 3-fold. Figure 9 represents the QC plot of arrays. 
Citation: Yadav R, Srivastava P (2016) Visualization of High Throughput Genomic Data Using R and Bioconductor. J Data Mining Genomics Proteomics 7: 197. doi:10.4172/2153-0602.1000197

Page 6 of 6

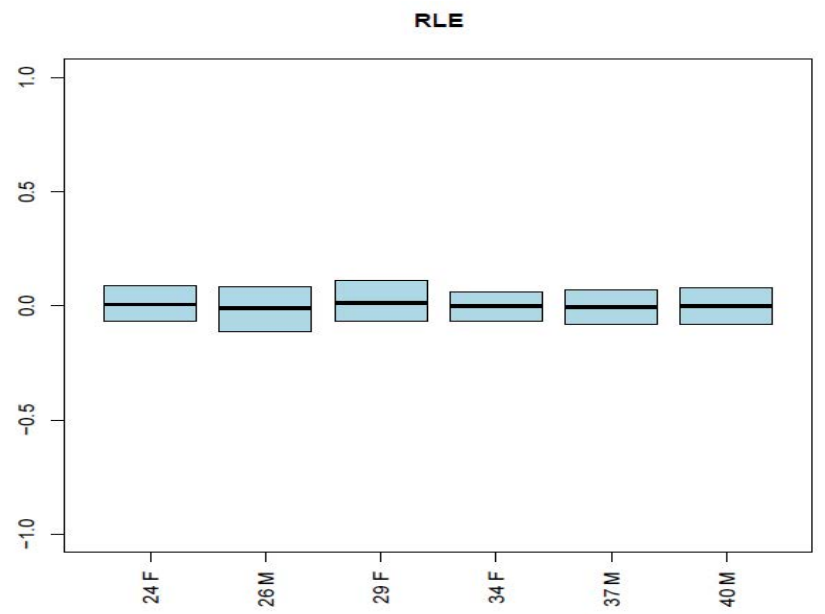

Figure 8: The variation in chips and used for quality assessment of probes.
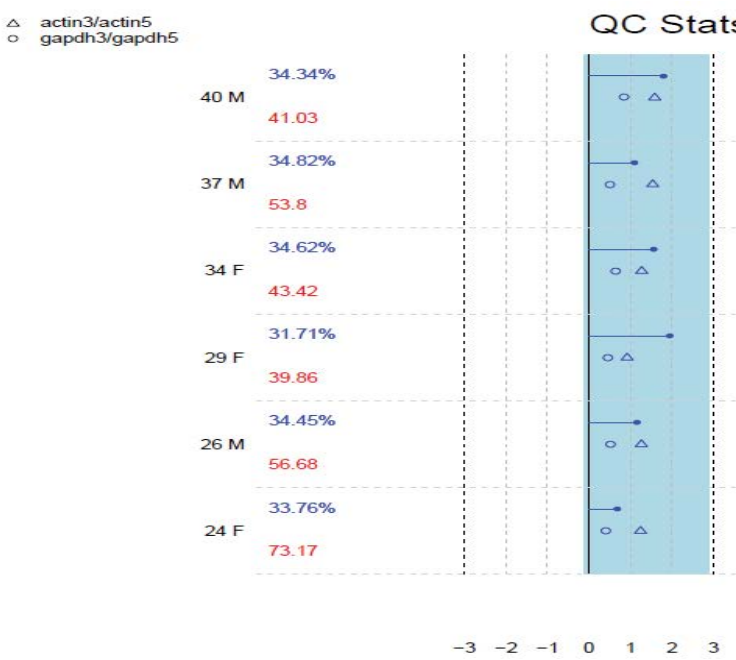

Figure 9: QC plot.

\section{Conclusion and Discussion}

This paper reviews the different quality control plots used for visualizing high throughput microarray data. And summarizes various packages of bioconductor that are used for microarray quality control and differentially expressed genes analysis. Any researcher working on microarray analysis can get help from this paper for microarray quality control analysis.

\section{Acknowledgements}

This is not just to follow the custom of writing acknowledgement but to express and record my heart felt feeling of thankfulness to all those who directly or indirectly helped me in this work. Further we wish to acknowledge bioinformatics tools for conducting this study.

\section{References}

1. Lu T, Aron L, Zullo J, Pan Y (2014) REST and stress resistance in ageing and Alzheimer's disease. Nature 507: 448-454.

2. Gautier L, Cope L, Bolstad BM, Irizarry RA (2004) Affy-analysis of Affymetrix GeneChip data at the probe level. Bioinformatics 20: 307-315.

3. Bolstad BM (2016) Affyio tools for parsing affymetrix data files. R package version 1: 40 .

4. http://www.bioconductor.org,http://bioinformatics.picr.man.ac.uk/simpleaffy/

5. Parman C, Halling C, Gentleman R (2008) QC Report Generation for affy Batch objects. $R$ package version 1.48.0.

6. Wu X, Li A (2009) ArrayTools: geneChip is Package. R package version 1.30.0.

7. Kauffmann A, Gentleman R, Huber W (2009) arrayQualityMetrics-a bioconductor package for quality assessment of microarray data. Bioinformatics pp: 415-416.

8. Kauffmann A, Rayner TF, Parkinson H, Kapushesky M, Lukk M, et al. (2009) Importing ArrayExpress datasets into R/Bioconductor. Bioinformatics 25: 2092 2094.

9. Ritchie ME, Phipson B, Wu D, Hu Y, Law CW, et al. (2015) limma powers differential expression analyses for RNA-sequencing and microarray studies. Nucleic Acids Research 43: 47

10. Talloen W, Verbeke T (2011) a4: Automated Affymetrix Array Analysis Umbrella Package. R package version 1.18.0.

11. Bengtsson H, Bullard J, Hansen K (2015) affxparser: Affymetrix File Parsing SDK. R package version 1.42.0.

12. Talloen W, Verbeke T, Casneuf T, Bondt AD, Osselaer S, et al. (2013) a4Base Automated Affymetrix Array Analysis Base Package.

13. Talloen W, Verbeke T (2011) a4Core: Automated Affymetrix Array Analysis Core Package.

14. Alloen W, Verbeke T (2011) a4Preproc: Automated Affymetrix Array Analysis Preprocessing Package.

15. Verbeke T (2010) a4Reporting: Automated Affymetrix Array Analysis Reporting Package.

16. Cope LM, Irizarry RA, Jaffee HA, Wu Z, Speed TP (2004) affycomp: Graphics Toolbox for Assessment of Affymetrix Expression Measures.

17. Smith CA (2010) annaffy: Annotation tools for Affymetrix.

18. Biological metadata (2013) $R$ package version 1.42.0.

19. Ates T (2011) Annmap: Genome annotation and visualisation package pertaining to Affymetrix arrays and NGS analysis.

20. Zacher B, Soeding J, Kuan PF, Siebert M, Tresch A (2010). Starr: Simple tiling array analysis of Affymetrix ChIP-chip data. BMC Bioinformatics 11: 194

21. http://www.ncbi.nlm.nih.gov/geo

22. https://www.bioconductor.org/

23. https://cran.r-project.org/ 